\title{
The Use of Positive Matrices for the Analysis of the Large Time Behavior of the Numerical Solution of Reaction-Diffusion Systems*
}

\author{
By Luciano Galeone
}

\begin{abstract}
In this paper we study the numerical solution of nonlinear reaction-diffusion systems with homogeneous Neumann boundary conditions, via the known $\theta$-method.

We show that if conditions for the positivity of solutions are imposed, then the study of the asymptotic behavior of the numerical solution can be done by means of the theory of stochastic matrices.

In this way it is shown that the numerical solution reproduces the asymptotic behavior of the corresponding theoretical one. In particular. we obtain the decay of the solution to its mean value.

An analysis of the asymptotic stability of the equilibrium points and the convergence of the numerical scheme is given based on the use of $M$-matrices.

Finally we consider the case in which the nonlinear term satisfies a condition of quasimonotonicity.
\end{abstract}

1. Introduction. Many applications in various fields necessitate the numerical solution of systems of $M$ nonlinear. reaction-diffusion equations:

$$
\frac{\partial U}{\partial t}=D \cdot \Delta U+F(U), \quad(x, t) \in \Omega \times R^{+} .
$$

where $\Omega \in R^{\nu}$ is a bounded domain with sufficiently smooth boundary and $\Delta$ is the Laplace operator in $R^{\nu}$. $D$ is a diagonal matrix whose elements are positive constants $d_{i}, i=1,2, \ldots, M$, and $F(U)=\left(f_{1}(U), f_{2}(U), \ldots, f_{M}(U)\right)$ is a smooth function.

The solution $U(x, t)=\left(U_{1}(x, t), U_{2}(x, t), \ldots, U_{M}(x, t)\right)$ of $(1.1 \mathrm{a})$ is subject to the initial conditions

$$
U(x, 0)=U_{0}(x) \text { for } x \text { in } \Omega,
$$

together with the homogeneous Neumann boundary conditions:

$$
\frac{\partial U}{\partial n}=0 \quad \text { on } \partial \Omega \times R,
$$

where $\partial U / \partial n$ is the outward normal derivative at a point $x \in \partial \Omega$.

Some examples of applications of the equations (1.1) can be found in the study of epidemics and many other biological topics, in ecology, chemistry and in problems dealing with the transmission of nerve pulses [6], [7].

Received December 16, 1981.

1980 Mathematics Subject Classification. Primary 65M10; Secondary 65C20.

Key words and phrases. Nonlinear reaction-diffusion systems, $\theta$ methods, $M$-matrices, stochastic matrices, $A$-stability, quasimonotone functions.

*Work performed in the context of Program of Preventive Medicine (Project MPPl), C.N.R., Italy. 
The boundary conditions (1.1c) indicate that the habitat $\Omega$ is isolated during the evolution of the system.

The physical meaning of solution $U$ demands that the numerical solution which is determined should be sufficiently able to simulate the behavior of the theoretical solution. Therefore we are most interested in the study of the behavior of the numerical solution and its asymptotic properties.

To this end, this paper describes some results by using a class of known methods ( $\theta$-methods). First of all we shall require the positivity of the numerical solution. This will allow us to use some results we can find in the study of compartmental systems and Markov chains [3]. Therefore, during our work we shall use mainly the properties of $M$-matrices and positive matrices.

The paper is organized as follows.

In Section 2 we introduce the known $\theta$-methods to discretize the system (1.1).

In Section 3 we observe that, if we require the positivity of the solution, then the matrices, which are in the discrete scheme, are stochastic. We therefore recall some properties of the stochastic matrices by using the group generalized inverse.

Thanks to these properties, in Section 4, we prove that, under suitable conditions, the numerical solution decays as time increases to a spatially homogeneous vector, which is its suitably weighted mean value. Such behavior reflects that of the theoretical solution, as shown in [5] and as studied in many other papers. We also analyze the case in which the numerical sequence converges towards a zero of the nonlinear term $F(U)$.

Finally, in the last section, we obtain certain results concerning the asymptotic stability for the equilibrium points of the discrete system and the asymptotic convergence of the numerical solution. We also derive an error estimate by imposing a monotonicity assumption on $F$.

In this paper we shall consider the parallelepiped $S=\prod_{i-{ }_{1}}^{M}\left[0, b_{i}\right]$ in $U$-space, and we shall suppose that $F$ is a smooth function which satisfies

$$
F(U) \cdot n_{S}(U) \leqslant 0 \text { for } U \text { in } \partial S,
$$

where $n_{S}(U)$ is the outward normal on $S$. Then, it is known [5] that $S$ is an invariant region for (1.1), that is, if $U_{0}(x) \in S$ for $x \in \Omega$, then, for $t>0, U(x, t)$ belongs to $S$.

We shall also assume that a constant $c$ exists, such that

$$
\left|\frac{\partial f_{i}}{\partial u_{i}}(U)\right| \leqslant c \quad \text { for } U \in S, i=1,2, \ldots, M .
$$

2. Finite Difference Approximation. For the discretization in the space variables, finite difference approximations or Galerkin procedures may be chosen [13], [14], whereas for the discretization of the resulting semidiscrete system in the time variable, we consider the well-known class of $\theta$-methods [13].

Let $h$ be an increment in the $x^{j}$ direction $(j=1,2, \ldots, \nu)$ and $\Delta t$ in the time direction. Let $x_{k}=\left(k_{1} h, k_{2} h, \ldots, k_{\nu} h\right)$ for $k \in Z^{\nu}$ and $t_{n}=n \Delta t$ for $n \in N$. If we put $I_{N}=\left\{k \in Z^{\nu} \mid x_{k} \in \Omega\right\}, x_{k}$ with $k \in I_{N}$ indicates a nodal point and $N$ the number of the nodal points. 
For any $\theta, 0 \leqslant \theta \leqslant 1$, and $\psi=1-\theta$, we approximate the solution of the system (1.1) at the time $t_{n+1}$ by means of the solution of the finite difference system:

$$
\left(I+\theta \frac{\Delta t}{h^{2}} d_{i} L\right) u_{i}^{n+1}=\left(I-\psi \frac{\Delta t}{h^{2}} d_{i} L\right) u_{i}^{n}+\Delta t f_{i}\left(u^{n}\right), \quad i=1,2, \ldots, M,
$$

where $I$ is the $N \times N$ identity matrix, $u_{i}^{n}$ and $f_{i}\left(u^{n}\right)$ are $N$-dimensional vectors, with elements $u_{i, k}^{n}, f_{i}\left(u_{1, k}^{n}, u_{2, k}^{n}, \ldots, u_{M, k}^{n}\right)$, for $k=1,2, \ldots, N$, respectively, and $u^{n}$ is the vector $\left(u_{1}^{n}, u_{2}^{n}, \ldots, u_{M}^{n}\right)^{\prime}$. The element $u_{i, k}^{n}$ is an approximation of the value $U\left(x_{k}, t_{n}\right)$.

In (2.1), the matrix $-L / h^{2}$ is a discrete operator, uniformly consistent with $\Delta$ in (1.1); that is, if $U(x, t)$ is a bounded function with bounded derivates up to second order and it satisfies (1.1c), then it is, for every $i$ :

$$
\sup _{k \in I_{N}}\left|-\frac{1}{h^{2}} L u_{i, k}^{n}-\Delta U_{i}\left(x_{k}, t_{n}\right)\right| \rightarrow 0 \quad \text { if } h \rightarrow 0 .
$$

It is known that the matrices $L=\left(L_{r s}\right)$, derived from the problem (1.1a), subject to the condition of $(1.1 \mathrm{c})$, have the following properties:

$$
L_{r r}>0, L_{r s} \leqslant 0 \quad \text { for } s \neq r \quad \text { and } \quad \sum_{s=1}^{N} L_{r s}=0, \quad \forall r=1,2, \ldots, N .
$$

Such matrices are irreducible with eigenvalues $0=\lambda_{1}<\lambda_{2} \leqslant \cdots \leqslant \lambda_{N}$. Some semidiscretization methods are such that the corresponding matrix $L$ is positive semidefinite.

Example 2.1. If we have $\nu=1$ and we use three-point difference approximation, $L$ is given by

$$
L=\left(\begin{array}{rrrrr}
2 & -2 & & & \\
-1 & 2 & & -1 & \\
& & \ddots & & \\
& & -1 & 2 & -1 \\
& & & -2 & 2
\end{array}\right)
$$

Instead, by using piecewise linear functions in the Galerkin procedure, we have

$$
L=\left(\begin{array}{rrrrr}
1 & -1 & & & \\
-1 & 2 & & -1 & \\
& & \ddots & & \\
& & -1 & 2 & -1 \\
& & & -1 & 1
\end{array}\right)
$$

which is a symmetric matrix.

It is known that there are $N$ linearly independent eigenvectors of the matrices $L$, given in the above examples. In the following, we shall suppose this for any matrix $L$.

In this paper we shall deal with square, nonnegative matrices, i.e. matrices $A=\left(A_{r s}\right)$ with $A_{r s} \geqslant 0$ for all $r, s$ and we denote $A \geqslant 0$. If $A_{r s}>0$ for all $r, s$ we shall put $A>0$.

Definition 2.1. A square matrix $A$ with $A_{r s} \leqslant 0$, for all $s \neq r$, is called an $M$-matrix if it is nonsingular and $A^{-1} \geqslant 0$. Moreover, it is called a singular $M$-matrix if it is singular and if, for all scalar $\varepsilon>0, A+\varepsilon I$ is an $M$-matrix. 
We recall the following known results [14]:

THEOREM 2.1. If $A$, with $A_{r s} \leqslant 0$ for all $s \neq r$, and $A_{r r}>0$ for all $r$, is an irreducible diagonally dominant matrix, then $A^{-1}>0$.

THEOREM 2.2. If $A$ is a nonnegative matrix, and $\rho(A)$ is its spectral radius, then $\rho(A)<1$, if and only if $(I-A)^{-1}$ exists and $(I-A)^{-1} \geqslant 0$.

Finally we observe that the matrix $L$ in (2.1), thanks to the properties (2.2), is a singular $M$-matrix.

3. Properties of the Difference Scheme. Let $L_{1}$ denote the $M N \times M N$ block-diagonal matrix, with block given by

$$
d_{i} \frac{\Delta t}{h^{2}} L, \quad i=1,2, \ldots, M
$$

and $I$ the $M N \times M N$ identity matrix. Then the system (2.1) takes the form

$$
\left(I+\theta L_{1}\right) u^{n+1}=\left(I-\psi L_{1}\right) u^{n}+\Delta t F\left(u^{n}\right),
$$

where $F\left(u^{n}\right)$ is the vector $\left(f_{1}\left(u^{n}\right), f_{2}\left(u^{n}\right), \ldots, f_{M}\left(u^{n}\right)\right)$.

It is known that, in the linear case $(F=0)$, the scheme is unconditionally stable if and only if $\frac{1}{2} \leqslant \theta \leqslant 1$. On the contrary, if $0 \leqslant \theta<\frac{1}{2}$, the scheme is stable only under a restriction on $\Delta t / h^{2}$ [13].

However, the usual stability conditions do not insure that the solution is positive and that the asymptotic behavior of the solution of the analytical problem is preserved for the solution of the scheme (3.1).

To avoid this, a stronger form of stability might be necessary [1], [9] such that the finite difference operator defined by (3.1) is of a positive type (i.e., if $u^{n}$ is nonnegative, then $u^{n+1}$ is also nonnegative).

In fact, we have the following theorem, where $\tilde{S}=\prod_{i=1}^{M}\left[0, b_{i}\right]^{N}$, and $c$ the constant given in (1.3):

THEOREM 3.1. Let us choose $0<\alpha<1$. If the following restrictions on the mesh

$$
\psi \frac{\Delta t}{h^{2}} \leqslant \frac{1-\alpha}{l}, \quad \Delta t<\frac{\alpha}{c},
$$

are satisfied, where $l=h^{2} \max _{1 \leqslant r \leqslant N M}\left(L_{1}\right)_{r r}$, then, if $u^{0} \in \tilde{S}$, for all $n>0$ the solution of (3.1) is nonnegative and belongs to $\tilde{S}$.

Proof. Thanks to the properties of the matrix $L,\left(I+\theta\left(\Delta t / h^{2}\right) d_{i} L\right)$, for all $i=1,2, \ldots, M$, is an irreducible $M$-matrix and Theorem 2.1 insures that it is $\left(I+\theta\left(\Delta t / h^{2}\right) d_{i} L\right)^{-1}>0$. From the first part of (3.2) we also have

$$
\left(I-\psi\left(\Delta t / h^{2}\right) d_{i} L\right) \geqslant 0 .
$$

Moreover, we observe that, if $u^{n} \in \tilde{S}, f_{i}\left(u^{n}\right) \geqslant-c u_{i}^{n}$. In fact, if we consider the vector $\tilde{u}^{n}=\left(u_{1}^{n}, \ldots, u_{i-1}^{n}, 0, u_{i+1}^{n}, \ldots, u_{M}^{n}\right)$, from the condition (1.2), we have $f_{i}\left(\tilde{u}^{n}\right)$ $\geqslant 0$ and then

$$
f_{i}\left(u^{n}\right)=f_{i}\left(\tilde{u}^{n}\right)+\left(\operatorname{diag} \frac{\partial f_{i}}{\partial u_{i}}\right) u_{i}^{n} \geqslant-c u_{i}^{n}
$$


It then follows that

$$
\left(I-\psi \frac{\Delta t}{h^{2}} d_{i} L\right) u_{i}^{n}+\Delta t f_{i}\left(u^{n}\right) \geqslant\left[(1-c \Delta t) I-\psi \frac{\Delta t}{h^{2}} d_{i} L\right] u_{i}^{n} \geqslant 0,
$$

and thus we obtain $u^{n+1} \geqslant 0$.

Similarly, we can see that $u^{n+1} \in \tilde{S}$ and prove the result of the theorem by induction.

We notice that if $\theta=1$ (implicit scheme), the first part of (3.2) is absent.

With the positivity assumption of the matrices in (3.1), we can now prove the following results:

THEOREM 3.2. If the restriction on the mesh

$$
\psi \frac{\Delta t}{h^{2}} \leqslant \frac{1}{l}
$$

is satisfied, then the matrix

$$
T_{i}=\left(I+\theta \frac{\Delta t}{h^{2}} d_{i} L\right)^{-1}\left(I-\psi \frac{\Delta t}{h^{2}} d_{i} L\right)
$$

is stochastic and primitive, for all $i=1,2, \ldots, M$.

Proof. From (2.2) it follows that the matrix $\left(I-\psi\left(\Delta t / h^{2}\right) d_{i} L\right)$ is stochastic. Moreover, it is $\rho\left(\left(I+\theta\left(\Delta t / h^{2}\right) d_{i} L\right)^{-1}\right)=1$, and since the vector $\xi$, whose elements are all one, is an eigenvector of $L$ associated with the null eigenvalue, we also have $\left(I+\theta\left(\Delta t / h^{2}\right) d_{i} L\right)^{-1} \xi=\xi$. Then $\left(I+\theta\left(\Delta t / h^{2}\right) d_{i} L\right)^{-1}$ is also stochastic.

Now, if $\theta \neq 0$, from $\left(I+\theta\left(\Delta t / h^{2}\right) d_{i} L\right)^{-1}>0$ it follows that $T_{i}>0$, and then $T_{i}$ is an irreducible and primitive matrix.

On the other hand, if $\theta=0$, it is $T_{i}=\left(I-\left(\Delta t / h^{2}\right) d_{i} L\right)$ and, since $T_{i}$ is irreducible and the diagonal elements of $T_{i}$ are positive, it is known that $T_{i}^{N-1}>0$.

Remark 3.1. We observe that

(i)

$$
\left(I+\theta \frac{\Delta t}{h^{2}} d_{i} L\right)^{\prime}\left(I-\psi \frac{\Delta t}{h^{2}} d_{i} L\right)=\left(I-\psi \frac{\Delta t}{h^{2}} d_{i} L\right)\left(I+\theta \frac{\Delta t}{h^{2}} d_{i} L\right)^{-1}
$$

(ii) if the matrix $L$ is symmetric, as for the example in (2.4), the matrix $T_{i}$ is doubly stochastic.

We recall now some results in the context of nonnegative irreducible matrices that are part of the classical Perron-Frobenius theorem.

THEOREM 3.3. If T is a nonnegative irreducible matrix, then

(i) $\rho(T)$ is a simple eigenvalue:

(ii) $T$ has a positive eigenvector corresponding to $\rho(T)$.

Then, if we consider a stochastic, primitive matrix $T$, since for every stochastic matrix $\rho(T)=1$, it is a simple eigenvalue. Hence, $\lambda=0$ is a simple eigenvalue of the matrix $A=I-T$ and $\operatorname{rank}(A)=\operatorname{rank}\left(A^{2}\right)$.

Now it is known [4] that the latter condition is equivalent to the existence of the group inverse of $A$; i.e. to that of the matrix $A^{D}$, such that

$$
A^{D} A A^{D}=A^{D}, \quad A A^{D}=A^{D} A \text { and } A A^{D} A=A .
$$


The properties of the matrix $A^{D}$ can be used to obtain classical results of the Markov chain theory [4].

For example, some known [10] fundamental results for regular (i.e., ergodic and noncyclic) Markov chains are given by

THEOREM 3.4. Let $T$ be the transition matrix for a regular Markov chain, and let $A=I-T$. Then

(a) $\lim _{k} T^{k}=R$, where $R=I-A A^{D}$;

(b) the matrix $R$ is a stationary stochastic matrix, i.e. each row of $R$ is given by the same probability vector. In particular, $R=\xi v^{T}$, where $v$ is the left eigenvector of $T$ associated with $\rho(T)=1$, such that $v^{T} \xi=1$;

(c) $T R=R T=R$ and $R^{2}=R$;

(d) if $p$ is an eigenvector of $T$, associated with $\rho(T)=1$, then $p$ is a constant vector;

(e) if $T$ is a doubly stochastic matrix, then it is $v=\xi / N$, where $N$ denotes the order of $T$.

Proof. The proofs of these results can also be found in [2], [4].

(a) It is known that a transition matrix $T$ for a regular chain is similar to a matrix of the form

$$
\left[\begin{array}{cc}
1 & 0 \\
0 & T_{1}
\end{array}\right],
$$

with $\lim _{k} T_{1}^{k}=0$. Then, it is easy to obtain the form of the matrix $A^{D}$ and to prove the result.

(b), (c) From the definition of $A^{D}$, it follows that $R A=A R=0$ and hence $T R=R T=R$. Then, each row of $R$ is a left eigenvector of $T$, associated with $\rho(T)=1$. Moreover, $R$ is stochastic, since $T^{k}$ is a stochastic matrix for all $k \in N$.

(d) If $T p=p$, then $T^{k} p=p$ and hence $R p=p$.

(e) The eigenvector $v$ is now a constant vector, such that $v^{T} \xi=1$.

On the other hand, it is known that if a stochastic matrix $T$ is the state transition matrix for a Markov chain, then $T$ is primitive, if and only if the chain is regular. Then, the result shown in the preceding theorem can be used for the primitive stochastic matrices $T_{i}$, given by (3.3), in order to study the large time behavior of the solution $u^{n}$ of (3.1).

As a first immediate consequence, we have the next result:

THEOREM 3.5. Let the hypotheses of Theorem 3.2 be satisfied. Let $T$ denote the $N M \times N M$ block-diagonal matrix

$$
T=\left(I+\theta L_{1}\right)^{-1}\left(I-\psi L_{1}\right)
$$

and $v$ the left eigenvector associated with the eigenvalue $\lambda_{1}=0$ of the matrix $L$.

Then $\lim _{k} T^{k}=R$, where $R$ is a block-diagonal matrix, with all equal blocks, given by $R_{1}=v^{T} \xi$.

Proof. From (i) in Remark 3.1, it follows directly, for all $i=1,2, \ldots, M$, that

$$
v^{T} T_{i}=v^{T} \Rightarrow v^{T} L=0 .
$$

Then, the proof is immediate from Theorem 3.4. 
The above theorem shows that the stationary matrix $R$ associated with the matrix $T$ is independent of the matrix $D$, of $h$ and $\Delta t$ and of the parameter $\theta$ in (2.1).

If we consider, for example, the matrix $L$ given by (2.3), we obtain

$$
v^{T}=\frac{1}{N-1}\left(\frac{1}{2}, 1, \ldots, 1, \frac{1}{2}\right)
$$

Instead, since the matrix $L$ given by (2.4) is symmetric, from (ii) in Remark 3.1 , and (e) in Theorem 3.4, it follows that

$$
v^{T}=\frac{1}{N}(1,1, \ldots, 1) .
$$

Finally, we observe that from the preceding results the next remark also follows.

Remark 3.2. Let the hypotheses of Theorem 3.2 be satisfied. Then the matrices

$$
\left(I+\theta \frac{\Delta t}{H} d_{i} L\right)^{-1} \text { and }\left(I-\psi \frac{\Delta t}{h} d_{i} L\right)
$$

for all $i=1,2, \ldots, M$, are stochastic and primitive, and the matrix $R_{1}$, defined in Theorem 3.5 , is the stationary matrix corresponding to them.

4. Large Time Behavior of the Numerical Solution. First of ali we observe that, for any vector $y$ of dimension $N$, the vector $R_{1} y$ has all its elements equal to $v^{T} y$. If $v$ is the vector given in (3.6), which we obtain when $L$ is symmetric, then the elements of $R_{1} y$ are the mean of $v$. On the other hand, if $v$ is the vector given in (3.5), the elements of $R_{1} y$ are a weighted mean of $y$, where the weight is due to the way in which the boundary conditions are discretized. Example 2.1 is given only for $\nu=1$, but a similar result is obtained when $\nu>1$.

The aim of the following theorem is to prove that, if

$$
\frac{d}{h^{2}} \lambda_{2}>k \cdot\|I-R\|
$$

holds true, where $\lambda_{2}$ is the smallest eigenvalue other than 0 of the matrix $L$, $d=\min _{1 \leqslant i \leqslant M}\left\{d_{i}\right\}$ and $k$ is the maximum of the norm of the Jacobian of $F$ for all points in $S$, then the difference between the numerical solution and its mean value (which might be "weighted") tends exponentially towards zero, as $t_{n}$ increases. The same type of result is proved in [8] by means of a different technique, using energy estimates.

The assumption (4.1) shows that the diffusion coefficient must be large relative to a quantity which measures the "strength" of the nonlinear interaction.

THEOREM 4.1. Let the hypotheses of Theorem 3.1 be satisfied and let the matrix $L$ be symmetric and positive semidefinite. If (4.1) is true, then we obtain for the solution $u^{n}$ of (3.1) that

$$
\left\|u^{n}-R u^{n}\right\| \leqslant(1-\Delta t q)^{n}\left\|u^{0}-R u^{0}\right\|
$$

where

$$
q=\frac{d \lambda_{2} / h^{2}-k\|I-R\|}{1+\theta\left(\Delta t / h^{2}\right) \lambda_{2} d}
$$


Proof. We observe that, for $i=1,2, \ldots, M$, the $N$ coordinates of the vector $f_{i}\left(R_{1} u_{1}^{n}, R_{1} u_{2}^{n}, \ldots, R_{1} u_{M}^{n}\right)$ are all equal. Therefore we have $f_{i}\left(R u^{n}\right)=R_{1} f_{i}\left(R u^{n}\right)$, that is,

$$
R F\left(R u^{n}\right)=F\left(R u^{n}\right)
$$

So it follows that

$$
(I-R) F\left(u^{n}\right)=(I-R)\left(F\left(u^{n}\right)-F\left(R u^{n}\right)\right)=(I-R) J_{F}\left(u^{n}-R u^{n}\right),
$$

where $J_{F}$ denotes the Jacobian matrix of $F$ evaluated at a point in $\tilde{S}$. Now, since it follows directly from Remark 3.2 that

$$
\left(I-\psi L_{1}\right) R=R\left(I-\psi L_{1}\right)=R, \quad\left(I+\theta L_{1}\right) R=R\left(I+\theta L_{1}\right)=R,
$$

we have, from (3.1),

$$
\left(I+\theta L_{1}\right)\left(u^{n+1}-R u^{n+1}\right)=\left(I-\psi L_{1}\right)\left(u^{n}-R u^{n}\right)+\Delta t(I-R) F\left(u^{n}\right)
$$

and hence, for (4.3),

$$
\begin{aligned}
\left(I+\theta L_{1}\right)\left(u^{n+1}-R u^{n+1}\right)= & \left(I-\psi L_{1}\right)\left(u^{n}-R u^{n}\right) \\
& +\Delta t(I-R) J_{F}\left(u^{n}-R u^{n}\right) .
\end{aligned}
$$

We denote the nonnegative eigenvalues of the matrix $L$ by $\lambda_{1}<\lambda_{2} \leqslant \cdots \leqslant \lambda_{N}$, and a system of orthonormal eigenvectors, which correspond to the eigenvalues $\mu_{k}=1$ $-\psi\left(\Delta t / h^{2}\right) d_{i} \lambda_{k}(k=1,2, \ldots, N)$ of the matrix $I-\psi\left(\Delta t / h^{2}\right) d_{i} L$, by $\left\{w_{k}^{i}\right\}$. We have $\mu_{1}^{i}=1$, and so we can take $w_{1}^{i}=\xi$ for all $i=1,2, \ldots, M$.

Therefore, for $u^{n}$, we can put $u_{i}^{n}=\sum_{k=1}^{N} \alpha_{k} w_{k}^{i}$, and so we obtain

$$
R_{1} u_{i}^{n}=\xi_{i} v^{T} \sum_{k=1}^{N} \alpha_{k} w_{k}^{i}=\alpha_{1} w_{1}^{i},
$$

because, for Remark 3.2, $v$ is the left eigenvector of $I-\psi\left(\Delta t / h^{2}\right) d_{i} L$ and it is $v^{T} \xi=1$.

On the other hand,

$$
\left(I-\psi \frac{\Delta t}{h^{2}} d_{i} L\right) u_{i}^{n}=\alpha_{1} w_{1}^{i}+\sum_{k=2}^{N} \alpha_{k} \mu_{k}^{i} w_{k}^{i},
$$

so that we obtain

$$
\left\|\left(I-\psi \frac{\Delta t}{h^{2}} d_{i} L\right)\left(u_{i}^{n}-R_{1} u_{i}^{n}\right)\right\| \leqslant \mu_{2}^{i}\left\|u_{i}^{n}-R_{1} u_{i}^{n}\right\| .
$$

In the same way we can also see

$$
\left\|\left(I+\theta \frac{\Delta t}{h^{2}} d_{i} L\right)\left(u_{i}^{n+1}-R_{1} u_{i}^{n+1}\right)\right\| \geqslant\left(I+\theta \frac{\Delta t}{h^{2}} d_{i} \lambda_{2}\right)\left\|u_{i}^{n+1}-R_{1} u_{i}^{n+1}\right\| .
$$

These inequalities together with (4.4) yield

$$
\left\|u^{n+1}-R u^{n+1}\right\| \leqslant \frac{1-\psi\left(\Delta t / h^{2}\right) d \lambda_{2}+\Delta t\|I-R\|\left\|J_{F}\right\|}{I+\theta\left(\Delta t / h^{2}\right) d \lambda_{2}}\left\|u^{n}-R u^{n}\right\|,
$$

from which the proof follows.

Remark 4.1. If the matrix $L$ is not symmetric the result of Theorem 5.1 is still true if there exists a nonsingular positive diagonal matrix $E$ such that $E \cdot L$ has a 
complete system of orthonormal eigenvectors. In this case the euclidean norm is substituted by the norm defined by the matrix $E$.

Remark 4.2. We would obtain a similar result if in the scheme (3.1) there were $F\left(u^{n+1}\right)$ instead of $F\left(u^{n}\right)$, that is, if we consider a nonlinear scheme.

Remark 4.3. By (3.1) and Remark 3.2 we have

$$
R u^{n+1}=R u^{n}+\Delta t R F\left(u^{n}\right) .
$$

If $F$ is a function which takes a nonpositive value in $S$, since $R u^{n} \geqslant 0$ for all $n$, the sequence $\left\{R u^{n}\right\}$ decreases to a limit. Under the hypotheses of the above theorem, the sequence $\left\{u^{n}\right\}$ is also convergent. In this case, if $u^{*}$ denotes the limit, we also have $F\left(u^{*}\right)=0$.

This is true even without the previous condition on $F$; in fact, from (4.2) it follows that

$$
R F\left(u^{n}\right)=F\left(R u^{n}\right)+R J_{F}\left(u^{n}-R u^{n}\right),
$$

and so (4.5) becomes

$$
R u^{n+1}=R u^{n}+\Delta t F\left(R u^{n}\right)+\Delta t R J_{F}\left(u^{n}-R u^{n}\right) .
$$

Therefore, if the hypotheses of Theorem 4.1 are true and the sequence $\left\{u^{n}\right\}$ converges, then it converges to a spatially homogeneous point $u^{*}$ such that $F\left(u^{*}\right)=0$.

Remark 4.4. According to Theorem 4.1, by (4.7), the vector $R u^{n}$ is the solution of the equation

$$
R u^{n+1}=R u^{n}+\Delta t F\left(R u^{n}\right)+g^{n},
$$

where $g^{\prime \prime}$ is a vector such that

$$
g_{i}^{n} \leqslant c_{1}(1-\Delta t q)^{n} \xi \text { for all } i=1,2, \ldots, M,
$$

with $c_{1}$ a positive constant. Therefore, for $n$ large enough, we can consider $R u^{n}$ and then $u^{n}$ as an approximation of the numerical solution of the ordinary differential equation $d V / d t=F(V)$.

Finally, let us suppose that a point $u^{*}$ exists such that $F\left(u^{*}\right)=0$. If $u^{*} \neq 0$, it must be $u^{*}=T u^{*}$, that is, $u$ is an eigenvector which corresponds to $\rho(T)=1$. Then, thanks to (d) in Theorem $3.4, u^{*}$ is a spatially homogeneous equilibrium point of (3.1), and, moreover, the only spatially homogeneous equilibrium points correspond to zeros of $F$. Therefore, Remark 4.3 says that, if the hypotheses in Theorem 4.1 are satisfied, the problem (3.1) has no nonconstant solutions in $S$.

5. Stability and Convergence. Let $\left\{u^{n}\right\}$ and $\left\{v^{n}\right\}$ be two sequences in $\tilde{S}$ given by (3.1); if we put $E_{n}=u^{n}-v^{n}$, it follows that

$$
E_{n+1}=\left(T+\Delta t\left(I+\theta L_{1}\right)^{-1} J_{F}\right) E_{n},
$$

where again $J_{F}$ denotes the Jacobian of $F$ for some points in $\tilde{S}$. Therefore, if

$$
\rho\left(T+\Delta t\left(I+\theta L_{1}\right)^{-1} J_{F}\right)<1
$$

for $J_{F}$ in $\tilde{S}$, both sequences tend to the same limit. So, if we consider $u^{*} \in \tilde{S}$, such that $F\left(u^{*}\right)=0$, which exists thanks to condition (1.2), $u^{*}$ is the unique asymptotically stable equilibrium point, since the sequence of constant value $u^{*}$ is a solution of (3.1). 
We denote by $\tilde{J}_{F}$ the Jacobian matrix in $S$. Then, if $-\tilde{J}_{F}$ in $S$ has positive diagonal elements and it is strictly diagonally dominant, that is, if a constant $\delta>0$ exists such that

$$
\left(-J_{F}\right)_{k k}-\sum_{j \neq k}\left|\left(-J_{F}\right)_{k j}\right|>\delta \text { for all } k
$$

then, if the hypotheses of Theorem 3.1 are satisfied, we have

$$
\|B\|_{\infty} \leqslant(1-\delta \Delta t)
$$

where $B=I-\Delta t\left(\psi L_{1}-J_{F}\right)$.

In fact, since $B$ has positive diagonal elements, from (2.2) it follows that

$$
\begin{aligned}
\|B\|_{\infty} & \leqslant \max _{k}\left\{1-\Delta t\left(\psi\left(L_{1}\right)_{k k}-\left(J_{F}\right)_{k k}\right)+\Delta t \sum_{j \neq k}\left(\left|\psi\left(L_{1}\right)_{k j}\right|+\left|\left(J_{F}\right)_{k j}\right|\right)\right\} \\
& =\max _{k}\left\{1-\Delta t\left[\left(-J_{F}\right)_{k k}+\sum_{j \neq k}\left|\left(J_{F}\right)_{k j}\right|\right]\right\} .
\end{aligned}
$$

In this case, we can derive an error bound between the exact solution of (1.1) and the approximant computed from (3.1), which is $O(\Delta t)$ with respect to time, uniformly in time.

Let $T^{n}$ denote the local truncation error, and assume that there is a constant $c_{2}$ such that $\left\|T^{n}\right\|_{\infty} \leqslant c_{2} \Delta t\left(\Delta t+h^{2}\right)$. Then we can prove the following theorem.

THEOREM 5.1. If the hypotheses of Theorem 3.1 and the additional assumption (5.3) on $F$ are satisfied, then

$$
\left\|E_{n}\right\|_{\infty} \leqslant(1-\delta \Delta t)^{n}\left\|E_{0}\right\|+\frac{c_{2}}{\delta}\left(\Delta t+h^{2}\right),
$$

where $E_{n}=u^{n}-U\left(t_{n}\right)$.

Proof. We observe that, from the properties of the matrix $L$,

$$
\left\|E_{n+1}\right\|_{\infty} \leqslant\|B\|_{\infty}\left\|E_{n}\right\|_{\infty}+\left\|T^{n}\right\|_{\infty} .
$$

Then we have

$$
\left\|E_{n+1}\right\|_{\infty} \leqslant(1-\delta \Delta t)\left\|E_{n}\right\|_{\infty}+c_{2} \Delta t\left(\Delta t+h^{2}\right),
$$

and the proof follows by induction.

In many applications the nonlinear term satisfies the following property [12]:

Definition 5.1. The function $F$ is said to be quasimonotone with respect to $u$ in $S$ if the matrix $\tilde{J}_{F}$ in $S$ has nonnegative off-diagonal elements.

In this case the following result holds.

THEOREM 5.2. Let $-\tilde{J}_{F}$ be an M-matrix in $S$, and let the hypotheses of Theorem 3.1 hold. Then the condition (5.2) is satisfied.

Proof. As observed at the end of Section 2, $L$ is a singular $M$-matrix, therefore, thanks to the hypotheses, the matrix $L_{1}-\Delta t J_{F}$ is an $M$-matrix too. Thus, the matrix

$$
C=I-\psi L_{1}+\Delta t J_{F}
$$

is such that $I-C$ is nonsingular and its inverse is positive. Since, using Theorem 3.1 , we have $C \geqslant 0$, the result follows from those of Theorem 2.2. 
Remark 5.2. Let the function $F$ be quasimonotone. Then, under the hypotheses of Theorem 3.1, the following implication holds: if $u^{0}, v^{0}$ belong to $\tilde{S}$ and $u^{0} \geqslant v^{0}$, then $u^{n} \geqslant v^{n}$ for all $n \in N$.

Now let us consider an arbitrary matrix $H$, and let $H^{+}$be the matrix obtained by replacing the off-diagonal elements of $H$ by their absolute values.

Then we have the following results:

THEOREM 5.3. Let the hypotheses of Theorem 3.1 be assumed true. If the matrix $-\tilde{J}_{F}^{+}$ is an M-matrix in $S$, then the condition (5.2) is satisfied.

Proof. We observe that, if we put

$$
\tilde{C}=I-\psi L_{1}+\Delta t J_{F}^{+}
$$

thanks to the previous theorem, we have $\rho(\tilde{C})<1$. Since we can see that $|C| \leqslant \tilde{C}$, then $\rho(|C|) \leqslant \rho(\tilde{C})$. Therefore, the result follows by observing that, for any matrix $C, \rho(C) \leqslant \rho(|C|)$.

Thanks to this result we can then use the theory of the $M$-matrices to have sufficient conditions for the asymptotic stability of the equilibrium points of the equations (3.1).

As an example, we give the following theorem, the proof of which can be found in [2].

THEOREM 5.4. Let $A$ be a matrix with nonpositive off-diagonal elements. Then the following propositions are equivalent:

(i) $A$ is an M-matrix.

(ii) $A$ has all positive diagonal elements, and there exists a diagonal matrix $D>0$ such that $D^{-1} A D$ is a strictly diagonally dominant matrix.

(iii) There exists a symmetric, definite positive matrix $W$, such that $A W+W A^{\prime}$ is definite positive.

(iv) The real part of each eigenvalue of $A$ is positive.

Therefore, for our purpose it is sufficient that the matrix $-\tilde{J}_{F}^{+}$satisfies one of the propositions of Theorem 5.3.

Finally, we observe that the condition on $-\tilde{J}_{F}$ required in Theorem 5.1 may be replaced by (ii) in Theorem 5.4 for $-\tilde{J}_{F}^{+}$. If a proposition in this theorem holds, then an error bound can be found analogous to the one shown in Theorem 5.1.

Istituto di Analisi Matematica

Università degli Studi di Bari

Palazzo Ateneo

Bari, Italy

1. C. Bolley \& M. Crouzeix, "Conservation de la positivité lors de la discrétisation des problèmes d'evolution paraboliques," RAIRO Anal. Numér., v. 12, 1978, pp. 237-245.

2. A. Berman \& R. Plemmons, Nonnegative Matrices in the Mathematical Sciences, Academic Press, New York, 1979.

3. V. Capasso \& S. L. Paveri-Fontana, "Some results on linear stochastic multicompartmental systems," Math. Biosci., v. 55, 1981, pp. 7-26.

4. S. L. CAMPBell \& C. D. MaYer, Generalized Inverses of Linear Transformations, Pitman, London, 1979.

5. E. Conway, D. Hoff \& J. SMOller, "Large time behavior of solutions of systems of nonlinear reaction-diffusion equations,” SIAM J. Appl. Math., v. 35, 1978, pp. 1-16. 
6. P. C. Fife, Mathematical Aspects of Reacting and Diffusing Systems, Lecture Notes in Biomath., Vol. 28, Springer-Verlag, New York, 1979.

7. W. E. Fitzgibbon \& H. F. Walker, Nonlinear Diffusion, Pitman, London, 1977.

8. L. GaleONE \& L. Lopez, "Decay to spatially homogeneous states for the numerical solution of reaction-diffusion systems," Calcolo, v. 19, 1982, pp. 193-208.

9. D. HofF, "Stability and convergence of finite difference methods for systems of nonlinear reaction-diffusion equations," SIAM J. Numer. Anal., v. 15, 1978, pp. 1161-1177.

10. J. G. Kemeny \& J. L. Snell, Finite Markot Chains, Springer-Verlag, New York, 1976.

11. J. P. LaSAlle, "Stability theory for difference equations," Studies in Ordinary Differential Equations, Math. Assoc. Amer., 1978.

12. R. H. Martin, "Asymptotic stability and critical points for nonlinear quasimonotone parabolic systems," J. Differential Equations, v. 30, 1978, pp. 301-423.

13. R. D. Richtmyer \& K. W. Morton, Difference Methods for Initial Value Problems, Interscience, New York, 1967.

14. R. S. Varga, Matrix Iterative Analysis, Prentice-Hall, Englewood Cliffs, N. J., 1962. 